\title{
ASYMPTOTIC CONTROLLABILITY AND EXPONENTIAL STABILIZATION OF NONLINEAR CONTROL SYSTEMS AT SINGULAR POINTS
}

\author{
LARS GRÜNE ${ }^{\dagger}$
}

\begin{abstract}
We discuss the relation between exponential stabilization and asymptotic controllability of nonlinear control systems with constrained control range at singular points. Using a discounted optimal control approach we construct discrete feedback laws minimizing the Lyapunov exponent of the linearization. Thus we obtain an equivalence result between uniform exponential controllability and uniform exponential stabilizability by means of a discrete feedback law.
\end{abstract}

Key words. stabilization, nonlinear control systems, singular points, Lyapunov exponents, discounted optimal control problems, discrete feedback control

AMS subject classifications. 93D15, 93D22

1. Introduction. In this paper we will present a technique for the exponential stabilization of nonlinear control systems with constrained control range at singular points. In particular we address the relation between asymptotic controllability and exponential stabilization and will derive an equivalence theorem. In our context a singular point is a fixed point for each admissible control value of the control system. Such singular situations do typically occur if the control enters in the parameters of an uncontrolled systems at a fixed point, for instance when the restoring force of a nonlinear oscillator is controlled. One example our results can be applied to is the stabilization problem of an inverted pendulum for which the suspension point is moved up an down periodically and the period of this motion can be controlled, cf. [14]. The main tool used throughout this paper is the linearization of the nonlinear system which forms a semilinear system. For two-dimensional control affine systems this linearization approach has been carried out in [4], giving a characterization of feedback stabilizability by algebraic methods.

The approach we follow here is based on optimal control techniques. More precisely, we consider the Lyapunov exponents of the linearization and formulate a discounted optimal control problem in order to minimize these exponents, an idea that has first been presented in [12]. Lyapunov exponents have recently turned out to be a suitable tool for the stability analysis of semilinear systems, see e.g. [7] and [8], and also for their stabilization [11]. However, due to the fact that for discounted optimal control problems optimal feedback laws are in general not available, we modify the feedback concept and introduce discrete feedback laws that are based on a discrete time sampled approximation of the given continuous time system. Using this approach it could be shown in [11] that for semilinear systems satisfying an accessibility condition exponential null controllability is equivalent to exponential stabilizability by discrete feedbacks. Using a similar feedback concept a result on the relation between asymptotic null controllability and practical stabilization for nonlinear systems has been developed in [5] using Lyapunov functions.

This paper is organized in two parts. In the first part we will focus on semilinear systems and extend the results from [11] and [12]. In particular in $\S 3$ we will

\footnotetext{
${ }^{\dagger}$ Institut für Mathematik, Universität Augsburg, Universitätsstr. 14, 86135 Augsburg, Germany, E-Mail: Lars.Gruene@Math.Uni-Augsburg.de. Research partially supported by DFG Grant Co 124/12-2
} 
discuss different null controllability concepts for semilinear systems and extend the approximation results from [12] to general semilinear systems without any accessibility assumptions. Then in $\$ 4$ we will use this result in order to construct a stabilizing discrete feedback law following the outline of [11].

In the second part we will apply this discrete feedback to a general nonlinear system at a singular point. For this purpose we will first prove a robustness property of the discrete feedback in $\$ 5$. Using this result we will present the main theorem in $\S 6$, stating that (local) uniform exponential null controllability is equivalent to (local) exponential stabilizability by means of a discrete feedback.

2. Preliminaries. We are interested in the stabilization of nonlinear control systems on $\mathbb{R}^{d} \times M$ given by

$$
\begin{aligned}
& \dot{x}(t)=f(x(t), y(t), u(t)) \\
& \dot{y}(t)=g(y(t), u(t))
\end{aligned}
$$

where $x \in \mathbb{R}^{d}$ and $y \in M, M$ some Riemannian Manifold and $f$ and $g$ are vectorfields which are $C^{2}$ in $x$, Lipschitz in $y$ and continuous in $u$. The control function $u(\cdot)$ may be chosen from the set $\mathcal{U}:=\{u: \mathbb{R} \rightarrow U \mid u(\cdot)$ measurable $\}$ where $U \subset \mathbb{R}^{m}$ is compact, i.e. we have a constrained set of control values.

For each pair $\left(x_{0}, y_{0}\right)$ of initial values the trajectories of $(2.1)$ will be denoted by the pair $\left(x\left(t, x_{0}, y_{0}, u(\cdot)\right), y\left(t, y_{0}, u(\cdot)\right)\right)$ and we assume them to exist uniquely for all times.

Our interest lies on the stabilization of the $x$-component at a singular point $x^{*}$, i.e. a point where $f\left(x^{*}, y, u\right)=0$ for all $(y, u) \in M \times U$. Throughout the paper we will assume $x^{*}=0$.

Note that our general setup covers several models: The additional equation for $y$ allows us to model systems where time varying parametric excitations governed by an additional (nonlinear) control or dynamical system enter the system to be stabilized. The case in which the control $u$ does not enter explicitly in the function $f$ and the case in which $f$ does not depend on $y$ occur as special situations in this setup, hence they are also covered.

Our main tool for the stabilization is the linearization of (2.1) at the singular point which is given by

$$
\begin{aligned}
& \dot{z}(t)=A(y(t), u(t)) z(t) \\
& \dot{y}(t)=g(y(t), u(t))
\end{aligned}
$$

Here $A(y, u):=\frac{\partial}{\partial x} f\left(x^{*}, y, u\right) \in \mathbb{R}^{d \times d}$ and $f(x, y, u)=A(y, u) x+\tilde{f}(x, y, u)$. Then for any given compact subset $K \subset M$ the differentiability assumption on $f$ implies the inequality

$$
\|\tilde{f}(x, y, u)\| \leq C_{f}\|x\|^{2}
$$

which holds for some constant $C_{f}$ for all $y \in K$ and all $x$ in a neighbourhood of $x^{*}$.

As above we denote the trajectories of $(2.2)$ by $\left(z\left(t, z_{0}, y_{0}, u(\cdot)\right), y\left(t, y_{0}, u(\cdot)\right)\right)$ for the pair of initial values $\left(z_{0}, y_{0}\right)$.

The first step is now to analyze and characterize the null controllability of (2.2).

3. Lyapunov exponents and their approximation. This section is concerned with the asymptotic null controllability of the semilinear system (2.2). From [7] it is known for bilinear systems that exponential null controllability of (2.2) can 
be characterized by certain Lyapunov exponents provided an accessibility condition holds and the matrix $A$ does not depend on $y$. These conditions will be dropped here and in addition we will show that the characterization is also valid if we replace exponential null controllability by asymptotic null controllability.

We will first introduce some concepts that will help us characterizing the properties of (2.2), see [6] and [7] for more details. Afterwards we will show the relation between different concepts of null controllability and then use these results in order to extend the approximation results from [12].

In order to measure the exponential null controllability we define the Lyapunov exponent of a trajectory of $(2.2)$ by

$$
\lambda\left(z_{0}, y_{0}, u(\cdot)\right):=\limsup _{t \rightarrow \infty} \frac{1}{t} \ln \| z\left(t, z_{0}, y_{0}, u(\cdot) \|\right.
$$

Clearly $\lambda\left(z_{0}, y_{0}, u(\cdot)\right)<0$ iff the corresponding trajectory converges to the origin exponentially fast. For each pair of initial values we define the infimal Lyapunov exponent by

$$
\lambda^{*}\left(z_{0}, y_{0}\right):=\inf _{u(\cdot) \in \mathcal{U}} \lambda\left(z_{0}, y_{0}, u(\cdot)\right)
$$

From the linearity of $(2.2)$ it follows that $\lambda\left(z_{0}, y_{0}, u(\cdot)\right)=\lambda\left(\alpha z_{0}, y_{0}, u(\cdot)\right)$ for all $\alpha \in \mathbb{R} \backslash\{0\}$. Hence we can use the projection of the $z$ component to the unit sphere $\mathbb{S}^{d-1}$ which is given by

$$
\begin{aligned}
& \dot{s}(t)=h(s(t), y(t), u(t)) \\
& \dot{y}(t)=g(y(t), u(t))
\end{aligned}
$$

where $h(s, y, u)=\left[A(y, u)-s^{T} A(y, u) s \mathrm{Id}\right] s$ where Id denotes the $d \times d$ identity matrix. Denoting the projected trajectory by $s\left(t, s_{0}, y_{0}, u(\cdot)\right)$ it follows from the chain rule that for $s_{0}=\frac{z_{n}}{\left\|z_{0}\right\|}$ the Lyapunov exponent can be written as

$$
\lambda\left(s_{0}, y_{0}, u(\cdot)\right)=\limsup _{t \rightarrow \infty} \frac{1}{t} \int_{0}^{t} q\left(s\left(\tau, s_{0}, y_{0}, u(\cdot)\right), y\left(\tau, y_{0}, u(\cdot)\right), u(\tau)\right) d \tau
$$

where $q(s, y, u):=s^{T} A(y, u) s$. This integral is also referred to as an averaged functional.

By defining the exponential growth rate in finite time $t$

$$
\lambda^{t}\left(z_{0}, y_{0}, u(\cdot)\right):=\frac{1}{t} \ln \frac{\| z\left(t, z_{0}, y_{0}, u(\cdot) \|\right.}{\left\|z_{0}\right\|}
$$

it is easily seen that

$$
\left\|z\left(t, z_{0}, y_{0}, u(\cdot)\right)\right\|=e^{t \lambda^{t}\left(z_{0}, y_{0}, u(\cdot)\right)}\left\|z_{0}\right\|
$$

As above this expression can be written in integral form using the projected system, i.e. for $s_{0}=\frac{z_{0}}{\left\|z_{0}\right\|}$ we obtain

$$
\lambda^{t}\left(s_{0}, y_{0}, u(\cdot)\right)=\frac{1}{t} \int_{0}^{t} q\left(s\left(\tau, s_{0}, y_{0}, u(\cdot)\right), y\left(\tau, y_{0}, u(\cdot)\right), u(\tau)\right) d \tau
$$

In our definitions of null controllability we need the notion of a positively invariant set for the subsystem on $M$. 
DEFINITION 3.1. A subset $K \subseteq M$ is called positively invariant for the subsystem of (2.D) on $M$ if for all $y_{0} \in K$ and all control functions $u(\cdot) \in \mathcal{U}$ the corresponding trajectory satisfies $y\left(t, y_{0}, u(\cdot)\right) \in K$ for all $t>0$.

Now we can define the concepts of null controllability, cp. also the stability concepts in $[15]$.

DEFINITION 3.2. Let $K \subseteq M$ be a compact positively invariant set for the subsystem of (2.2) on $M$.

(i) The system (D.D) is called asymptotically null controllable over $K$ if for any pair of initial values $\left(z_{0}, y_{0}\right) \in \mathbb{R}^{d} \times K$ there exists a control function $u(\cdot) \in \mathcal{U}$ such that

$$
\lim _{t \rightarrow 0}\left\|z\left(t, z_{0}, y_{0}, u(\cdot)\right)\right\|=0
$$

(ii) The system (D.D) is called exponentially null controllable over $K$ if $\lambda^{*}$ satisfies $\sup _{\left(z_{0}, y_{0}\right) \in \mathbb{R}^{d} \times K} \lambda^{*}\left(z_{0}, y_{0}\right)<0$

(iii) The system (2.2) is called uniformly exponentially null controllable over $K$ if there exist constants $C, \alpha>0$, such that for any pair of initial values $\left(z_{0}, y_{0}\right) \in \mathbb{R}^{d} \times K$ there exists a control function $u_{\left(z_{0}, y_{0}\right)}(\cdot) \in \mathcal{U}$ with

$$
\left\|z\left(t, z_{0}, y_{0}, u_{\left(z_{0}, y_{0}\right)}(\cdot)\right)\right\| \leq C e^{-\alpha t}\left\|z_{0}\right\|
$$

An immediate consequence from (3.3) is that (2.2) is uniformly exponentially null controllable over $K$ iff there exists a time $T>0$ and a constant $\sigma<0$ such that for any pair of initial values $\left(z_{0}, y_{0}\right) \in \mathbb{R}^{d} \times K$ there exists a control function $u_{\left(z_{0}, y_{0}\right)}(\cdot) \in \mathcal{U}$ with

$$
\lambda^{t}\left(z_{0}, y_{0}, u_{\left(z_{0}, y_{0}\right)}(\cdot)\right) \leq \sigma<0
$$

for all $t \geq T$.

It is easily seen from this definition that (iii) $\Rightarrow$ (ii) $\Rightarrow$ (i). In fact also the converse is true, i.e. the definitions are equivalent as the following proposition shows.

Proposition 3.3. Let $K \subseteq M$ be a compact positively invariant set for the subsystem of (2.2) on $M$. Then for the system (2.2) asymptotic null controllability over $K$ implies uniform exponential null controllability over $K$.

Proof. We will first show the following property: There exist $T>0$ and $\sigma<0$ such that for each $(z, y) \in \mathbb{R}^{d} \times K$ there exists a control function $u_{(z, y)}(\cdot) \in \mathcal{U}$ and a time $t_{(z, y)} \leq T$ such that $\lambda^{t_{(z, y)}}\left(z, y, u_{(z, y)}(\cdot)\right)<\sigma$.

The asymptotic null controllability implies, that for each $\left(\tilde{z}_{0}, \tilde{y}_{0}\right) \in \mathbb{R}^{d} \times K$ there exists a time $\tilde{t}_{\left(\tilde{z}_{0}, \tilde{y}_{0}\right)}$ and a control function $\tilde{u}_{\left(\tilde{z}_{0}, \tilde{y}_{0}\right)}(\cdot)$, such that

$$
\left\|z\left(\tilde{t}_{\left(\tilde{z}_{0}, \tilde{y}_{0}\right)}, \tilde{z}_{0}, \tilde{y}_{0}, \tilde{u}_{\left(\tilde{z}_{0}, \tilde{y}_{0}\right)}(\cdot)\right)\right\|<\frac{1}{3}\left\|\tilde{z}_{0}\right\|
$$

Considering only those $\tilde{z}_{0}$ with $\left\|\tilde{z}_{0}\right\|=1$ (i.e. $\tilde{z}_{0} \in \mathbb{S}^{d-1}$ ) and using the continuous dependence on the initial value we find a neighbourhood $U\left(\tilde{z}_{0}, \tilde{y}_{0}\right)$ in $\mathbb{S}^{d-1} \times K$ such that for each $(z, y) \in U\left(\tilde{z}_{0}, \tilde{y}_{0}\right)$ it holds that

$$
\left\|z\left(\tilde{t}_{\left(\tilde{z}_{0}, \tilde{y}_{0}\right)}, z, y, \tilde{u}_{\left(\tilde{z}_{0}, \tilde{y}_{0}\right)}(\cdot)\right)\right\|<\frac{1}{2}\|z\| .
$$


Hence it follows that $\lambda^{\tilde{t}_{\left(\tilde{z}_{0}, \tilde{y}_{0}\right)}}\left(z, y, \tilde{u}_{\left(\tilde{z}_{0}, \tilde{y}_{0}\right)}(\cdot)\right)<\gamma_{\left(\tilde{z}_{0}, \tilde{y}_{0}\right)}<0$ where

$$
\gamma_{\left(\tilde{z}_{0}, \tilde{y}_{0}\right)}=\frac{\ln \frac{1}{2}}{\tilde{t}_{\left(\tilde{z}_{0}, \tilde{y}_{0}\right)}}
$$

By the compactness of $\mathbb{S}^{d-1} \times K$ we may pick a finite number of pairs $\left(\tilde{z}_{0}, \tilde{y}_{0}\right)$ such that the neighbourhoods $U\left(\tilde{z}_{0}, \tilde{y}_{0}\right)$ cover $\mathbb{S}^{d-1} \times K$. Now the independence of $\lambda^{t}$ from the norm of $z$ yields the asserted property where $T$ is the maximum over all $\tilde{t}_{\left(\tilde{z}_{0}, \tilde{y}_{0}\right)}$ and $\sigma<0$ the maximum over all $\gamma_{\left(\tilde{z}_{0}, \tilde{y}_{0}\right)}$.

Now pick an arbitrary pair $\left(z_{0}, y_{0}\right)$ of initial values. We use the control $u_{0}(\cdot):=$ $u_{\left(z_{0}, y_{0}\right)}(\cdot)$ from above up to the time $t_{1}:=t_{\left(z_{0}, y_{0}\right)}<T$ from above and end up at the point $\left(z_{1}, y_{1}\right)=\left(z\left(t_{1}, z_{0}, y_{0}, u_{0}(\cdot)\right), y\left(t_{0}, y_{0}, u_{0}(\cdot)\right)\right.$. We continue iteratively by defining $t_{i+1}:=t_{i}+t_{\left(z_{i}, y_{i}\right)}$ and $u_{i}(\cdot):=u_{\left(z_{i}, y_{i}\right)}(\cdot)$ and define a control function $u: \mathbb{R}^{+} \rightarrow U$ by

$$
u(t):=u_{i}\left(t-t_{i}\right), t \in\left[t_{i}, t_{i+1}\right]
$$

for $i \in \mathbb{N}_{0}$ where $t_{0}:=0$.

This yields $\lambda^{t_{i}}\left(z_{0}, y_{0}, u(\cdot)\right)<\sigma$ for all $t_{i}, i \in \mathbb{N}_{0}$ and since $t_{i}-t_{i-1}<T$ it follows that for any $t>0$ there exists $t_{i}=: t_{i}(t)$ with $0 \leq t-t_{i}(t)<T$. By the definition of $\lambda^{t}$ we obtain

$$
\lambda^{t}\left(z_{0}, y_{0}, u(\cdot)\right)=\frac{t_{i}(t)}{t} \lambda^{t_{i}(t)}\left(z_{0}, y_{0}, u(\cdot)\right)+\frac{t-t_{i}(t)}{t} \lambda^{t-t_{i}(t)}\left(z_{i}, y_{i}, u_{i}(\cdot)\right)
$$

which yields

$$
\lambda^{t}\left(z_{0}, y_{0}, u(\cdot)\right)<\sigma+\varepsilon(t)
$$

where

$$
\varepsilon(t)=\frac{t-t_{i}(t)}{t}\left(\lambda^{t-t_{i}(t)}\left(z_{i}, y_{i}, u_{i}(\cdot)\right)-\lambda^{t_{i}(t)}\left(z_{0}, y_{0}, u(\cdot)\right)\right)
$$

implying $\varepsilon(t) \rightarrow 0$ for $t \rightarrow \infty$ independently from $\left(z_{0}, y_{0}\right)$ since $\lambda^{t}$ is uniformly bounded for all $t>0$ and all $(z, y) \in \mathbb{R}^{d} \times K$. Hence there exists $\varepsilon>0$ and a time $T>0$ such that $\lambda^{t}\left(z_{0}, y_{0}, u(\cdot)\right)<\sigma+\varepsilon<0$ for all $t \geq T$ and the assertion follows. $\square$

Using essentially the same arguments as in the previous proof, we can also determine the uniform upper bound for the values of the $\lambda^{t}$.

Proposition 3.4. Let $K \subseteq M$ be a compact positively invariant set for the subsystem of (2.2) on $M$. Let $\sigma:=\sup _{\left(z_{0}, y_{0}\right) \in \mathbb{R}^{d} \times K} \lambda^{*}\left(z_{0}, y_{0}\right)$.

Then for each $\varepsilon>0$ there exists a $T>0$ such that for any $\left(z_{0}, y_{0}\right) \in \mathbb{R}^{d} \times K$ there exists a control function $u(\cdot) \in \mathcal{U}$ satisfying

$$
\lambda^{t}\left(z_{0}, y_{0}, u(\cdot)\right)<\sigma+\varepsilon
$$

for all $t \geq T$.

Proof. For any pair $\left(\tilde{z}_{0}, \tilde{y}_{0}\right) \in \mathbb{R}^{d} \times K$ there exists a control function $\tilde{u}_{\left(\tilde{z}_{0}, \tilde{y}_{0}\right)}(\cdot)$ and a time $\tilde{t}_{\left(\tilde{z}_{0}, \tilde{y}_{0}\right)}$ such that

$$
\lambda^{\tilde{t}_{\left(\tilde{z}_{0}, \tilde{y}_{0}\right)}}\left(\tilde{z}_{0}, \tilde{y}_{0}, \tilde{u}_{\left(\tilde{z}_{0}, \tilde{y}_{0}\right)}(\cdot)\right)<\sigma+\frac{\varepsilon}{3}
$$


As in the previous proof continuous dependence and compactness implies that for any pair $(z, y)$ there exist $t_{(z, y)}$ bounded by some $\tilde{T}$ and control functions $u_{(z, y)} \in \mathcal{U}$, such that

$$
\lambda^{t_{(z, y)}}\left(z, y, u_{(z, y)}(\cdot)\right)<\sigma+\frac{\varepsilon}{2}
$$

Following the previous proof we can iteratively construct control functions satisfying

$$
\lambda^{t}\left(z_{0}, y_{0}, u(\cdot)\right)<\sigma+\frac{\varepsilon}{2}+\varepsilon(t)
$$

Again $\varepsilon(t)$ can be chosen independently from $\left(z_{0}, y_{0}\right)$ and $\varepsilon(t) \rightarrow 0$ as $t \rightarrow \infty$; hence the assertion follows by choosing $T$ such that $\varepsilon(t)<\frac{\varepsilon}{2}$ for all $t \geq T$. $\mathbf{\square}$

This result implies that the $\alpha$ in Definition 3.2 (iii) can be chosen arbitrarily close to the sup-inf Lyapunov exponent $\sigma$ as defined in Proposition 3.4. This Lyapunov exponent therefore gives the characteristic value for the null controllability of (2.2).

The construction of the stabilizing discrete feedback in the next section - following the outline of [11] — is based on the minimization of the Lyapunov exponent. This is related to minimizing (3.2) which forms an average time optimal control problem, for which the construction of optimal feedback controls is still an unsolved problem.

Hence we will not approach this problem directly but we will use the approximation of (3.2) by a discounted functional with discount rate $\delta>0$ defined by

$$
J_{\delta}\left(s_{0}, y_{0}, u(\cdot)\right):=\int_{0}^{\infty} e^{-\delta \tau} q\left(s\left(\tau, s_{0}, y_{0}, u(\cdot)\right), y\left(\tau, y_{0}, u(\cdot)\right), u(\tau)\right) d \tau
$$

The function

$$
v_{\delta}\left(s_{0}, y_{0}\right):=\inf _{u(\cdot) \in \mathcal{U}} J_{\delta}\left(s_{0}, y_{0}, u(\cdot)\right)
$$

is called the optimal value function of this discounted optimal control problem.

The relation between this problem and the minimization of (3.2) has been discussed in [12] for the case where (3.1) is locally accessible exploiting the controllability properties of (3.1). Here we will use Proposition 3.4 in combination with a stronger version of the Approximation Theorems from [12] in order to show this relation without assuming local accessibility.

Lemma 3.5. (Approximation Theorems) Let $q: \mathbb{R} \rightarrow \mathbb{R}$ be a measurable function satisfying $|q(s)|<M_{q}$ for almost all $s \in \mathbb{R}$.

(i) Assume there exists a time $T>0$ such that

$$
\frac{1}{t} \int_{0}^{t} q(\tau) d \tau<\sigma \text { for all } t \geq T
$$

Then for any $\varepsilon>0$ and all $0<\delta<\frac{\varepsilon}{\left(M_{q}+\sigma+\varepsilon\right) T}$ the following inequality holds:

$$
\delta \int_{0}^{\infty} e^{-\delta \tau} q(\tau) d \tau \leq \sigma+\varepsilon
$$

(ii) Let $\delta>0$ be arbitrary and let

$$
\delta \int_{0}^{\infty} e^{-\delta \tau} q(\tau) d \tau=: \sigma
$$


Then for any $\varepsilon>0$ there exists a $T \in\left[\frac{\varepsilon}{\left(4 M_{q}+4 \sigma+\varepsilon\right) \delta},-\frac{1}{\delta} \ln \frac{\varepsilon}{4 M_{q}}\right]$ satisfying

$$
\frac{1}{T} \int_{0}^{T} q(\tau) d \tau \leq \sigma+\varepsilon
$$

(iii) Let $\delta>0$ be arbitrary and let $\sigma \in \mathbb{R}$ such that

$$
\delta \int_{0}^{\infty} e^{-\delta \tau} q(t+\tau) d \tau \leq \sigma \text { for all } t \geq 0
$$

Then

$$
\limsup _{T \rightarrow \infty} \frac{1}{T} \int_{0}^{T} q(\tau) d \tau \leq \sigma
$$

Proof. The rather technical proof can be found in the appendix.

Next we can formulate the consequence for the optimal value function.

THEOREM 3.6. Let $K \subseteq M$ be a compact positively invariant set for the subsystem on $M$ of (2.2). Then

$$
\lim _{\delta \rightarrow 0} \sup _{(s, y) \in \mathbb{S}^{d-1} \times K} \delta v_{\delta}(s, y)=\sup _{(s, y) \in \mathbb{S}^{d-1} \times K} \lambda^{*}(s, y)
$$

Proof. Let $\sigma:=\sup _{(s, y) \in \mathbb{S}^{d-1} \times K} \lambda^{*}(s, y)$ and $\varepsilon>0$. By Proposition 3.4 there exists a time $T>0$ such that for each pair $(s, y) \in \mathbb{S}^{d-1} \times K$ there exists a control function $u(\cdot) \in \mathcal{U}$ such that

$$
\lambda^{t}(s, y, u(\cdot))<\sigma+\frac{\varepsilon}{2}
$$

By Lemma 3.5 (i) this implies

$$
\delta J_{\delta}(s, y, u(\cdot))<\sigma+\varepsilon
$$

for all sufficiently small $\delta>0$. Since $\varepsilon>0$ was arbitrary this implies

$$
\limsup _{\delta \rightarrow 0} \sup _{(s, y) \in \mathbb{S}^{d-1} \times K} \delta v_{\delta}(s, y) \leq \sigma .
$$

Now assume $\liminf \operatorname{in}_{\delta \rightarrow 0} \sup _{(s, y) \in \mathbb{S}^{d-1} \times K} \delta v_{\delta}(s, y)=\gamma<\sigma$. Then there exists $\delta>0$ such that by Bellman's optimality principle [19, Theorem 1.2] for each pair $(s, y)$ there exists a control function $u(\cdot)$ satisfying

$$
\delta J_{\delta}(s(t, s, y, u(\cdot)), y(t, y, u(\cdot)), u(t+\cdot))<\tilde{\gamma}<\sigma
$$

for all $t \geq 0$. Now by Lemma 3.5 (iii) it follows that $\lambda^{*}(s, y) \leq \tilde{\gamma}<\sigma$ which contradicts the definition of $\sigma$. Hence the assertion follows. $\square$

This theorem states that the Lyapunov exponent that gives the characteristic number for null controllability can be approximated by the value function of a discounted optimal control problem.

Since algorithms for the numerical computation of $v_{\delta}$ are known (cf. e.g. [9] and [13]) this theorem also lays the foundation for the numerical null controllability analysis of semilinear systems, see also [12]. This is of particular interest because the question whether $(2.2)$ is null controllable can in general not by answered by analytical methods. 
4. Construction of the discrete feedback. We will now present a feedback construction for the (approximately) optimal solution of the discounted optimal control problem defined by (3.4) and (3.5), which will then be stabilizing for (2.2).

In general the construction of optimal feedback laws for discounted optimal control problems is an unsolved problem. One of the main problems is that optimal feedbacks are typically discontinuous and hence properties like the existence and uniqueness of the corresponding solutions are no longer guaranteed. Some effort has been made in order to take these difficulties into account e.g. by using differential inclusions (see [10] and [1]). However, apart from the fact that this approach leads to a characterization of optimal trajectories rather than to a construction of a feedback law, from the stabilization (and application) point of view it seems desirable to preserve these properties. Furthermore we will need a certain robustness property as discussed in $\S 5$ in order to apply the feedback to the nonlinear system.

These considerations lead to a somewhat modified feedback concept which is based on an approximation of $\mathcal{U}$ as introduced in [11]. Theorem 3.6 yields the property needed for the construction of the stabilizing discrete feedback in $\S \S 3$ and 4 of this reference and our construction now follows this outline. We will therefore just give the idea of the construction and omit the proofs except for the concluding theorem.

We approximate $\mathcal{U}$ by

$$
\mathcal{U}_{h}:=\left\{u: \mathbb{R} \rightarrow U|u|_{[i h,(i+1) h)} \equiv u_{i} \text { for all } i \in \mathbb{Z}\right\}
$$

for some time step $h>0$. This discretization for discounted optimal control problems bears some similarity to the discretization in [2] and [3]; in fact what we obtain is a discrete time system by the process of sampling (cf. [21, Section 2.10]):

$$
s_{i+1}=s\left(h, s_{i}, y_{i}, u_{i}\right), y_{i+1}=y\left(h, y_{i}, u_{i}\right)
$$

where $\left(u_{i}\right)_{i \in \mathbb{Z}} \in U^{\mathbb{R}}$.

Defining

$$
v_{\delta}^{h}\left(s_{0}, y_{0}\right):=\inf _{u(\cdot) \in \mathcal{U}_{h}} J_{\delta}\left(s_{0}, y_{0}, u(\cdot)\right)
$$

the approximation property

$$
\left\|v_{\delta}-v_{\delta}^{h}\right\|_{\infty} \leq C h^{\frac{\gamma}{2}}
$$

holds for $\gamma=\delta / L$ where $L$ denotes the Lipschitz constant of (3.1), see [3].

Bellman's optimality principle [19, Theorem 1.2$]$ yields

$$
\begin{aligned}
& v_{\delta}^{h}\left(s_{0}, y_{0}\right)= \\
& \inf _{u \in U}\left\{\int_{0}^{h} e^{-\delta \tau} q\left(s\left(\tau, s_{0}, y_{0}, u\right), y\left(\tau, y_{0}, u\right), u\right) d \tau+e^{-\delta h} v_{\delta}^{h}\left(s\left(h, s_{0}, y_{0}, u\right), y\left(h, y_{0}, u\right)\right)\right\}
\end{aligned}
$$

By the continuity of all functions involved and the compactness of $U$ we can now define a function $F: \mathbb{S}^{d-1} \times K \rightarrow U$ by choosing $F\left(s_{0}, y_{0}\right):=u \in U$ such that the infimum above is attained in $u$.

We may now apply $F$ to (3.1) by

$$
\begin{aligned}
& \dot{s}(t)=h\left(s(t), y(t), F\left(s\left(\left[\frac{t}{h}\right] h\right), y\left(\left[\frac{t}{h}\right] h\right)\right)\right) \\
& \dot{y}(t)=g\left(y(t), F\left(s\left(\left[\frac{t}{h}\right] h\right), y\left(\left[\frac{t}{h}\right] h\right)\right)\right)
\end{aligned}
$$


We denote the solution trajectories of (4.2) by $\left(s_{F}\left(t, s_{0}, y_{0}\right), y_{F}\left(t, y_{0}\right)\right)$.

Feedback laws of this kind can be found in the literature under the name of modified feedback control [16], [17], sample-and-hold control or sampled feedback [20], [22] and step-by-step control [18]. Of particular interest in this context is the recent work [5] where a stabilization result using a "sampled feedback" control is presented. We will discuss the relation between this work and the present paper in $\S 6$.

In our terminology we call $F$ a "discrete" feedback control, a notion being motivated by the fact that $F$ is indeed a feedback control for the discrete time system (4.1). From this interpretation the existence and uniqueness of the trajectories of (4.2) is immediately clear.

If we evaluate

$$
J_{\delta}(s, y, F):=\int_{0}^{\infty} e^{-\delta \tau} q\left(s_{F}(\tau, s, y), y_{F}(\tau, y), F\left(s_{F}\left(\left[\frac{\tau}{h}\right] h, s, y\right), y_{F}\left(\left[\frac{\tau}{h}\right] h, y\right) \tau\right)\right) d \tau
$$

i.e. the discounted value along the trajectories of (4.2) it follows that $J_{\delta}(s, y, F)=$ $v_{\delta}^{h}(s, y)$ for all initial values $(s, y) \in \mathbb{S} \times K$ ([11, Theorem 3.6]). Hence $F$ forms an optimal discrete feedback for the discounted optimal control problem with respect to the discretized control functions from $\mathcal{U}_{h}$.

In the same way we define the averaged value along the trajectories by

$$
\lambda^{t}(s, y, F):=\frac{1}{t} \int_{0}^{t} q\left(s_{F}(\tau, s, y), y_{F}(\tau, y), F\left(s_{F}\left(\left[\frac{\tau}{h}\right] h, s, y\right), y_{F}\left(\left[\frac{\tau}{h}\right] h, y\right) \tau\right)\right) d \tau
$$

By defining $F_{\mathbb{R}}(z, y):=F(z /\|z\|, y)$ we can apply $F_{\mathbb{R}}$ to the non projected system

$$
\begin{aligned}
& \dot{z}(t)=A\left(y(t), F_{\mathbb{R}}\left(s\left(\left[\frac{t}{h}\right] h\right), y\left(\left[\frac{t}{h}\right] h\right)\right)\right) z(t) \\
& \dot{y}(t)=g\left(y(t), F_{\mathbb{R}}\left(z\left(\left[\frac{t}{h}\right] h\right), y\left(\left[\frac{t}{h}\right] h\right)\right)\right)
\end{aligned}
$$

As above we denote the corresponding trajectories by $\left(x_{F_{\mathbb{R}}}\left(t, x_{0}, y_{0}\right), y_{F_{\mathbb{R}}}\left(t, y_{0}\right)\right)$. Applying $F_{\mathbb{R}}$ this way we can state the following theorem.

THEOREM 4.1. Let $K \subset M$ be a compact positively invariant set for the subsystem of (2.2) on $M$. Then (2.2) is asymptotically null controllable over $K$ if and only if there exists a time step $h$ and a discrete feedback law $F_{\mathbb{R}}: \mathbb{R} \times K \rightarrow U$ such that (4.3) is uniformly exponentially stable, i.e. there exists $C, \alpha>0$ such that every trajectory of (4.3) satisfies the condition from Definition 3.2 (iii).

Proof. “ $\Rightarrow$ ” Assume asymptotic null controllability of (2.2). By [11, Corollary $3.7]$, it follows that for any $\varepsilon>0$ there exists $h>0$ such that the discrete feedback as defined above satisfies

$$
\delta J_{\delta}(s, y, F)<\delta v_{\delta}(s, y)+\varepsilon
$$

Choosing $\delta>0$ sufficiently small Proposition 3.3 implies that there exists $\sigma<0$ such that $\delta J_{\delta}(s, y, F)<\sigma$, hence from Lemma 3.5 (ii) we can conclude that for any $\varepsilon>0$ there exists a bounded time $t=t(\varepsilon)>0$ such that $\lambda^{t}(s, y, F)<\sigma+\varepsilon$. Using [11, Lemma 4.1] we obtain estimate (4.4) for the next trajectory piece and can inductively obtain the assertion as in the proof of Proposition 3.3.

" $\Leftarrow$ " This direction is immediately clear.

Note that this stabilizing discrete feedback law is numerically computable - at least for lower dimensional systems — using the algorithm proposed in [11] and [13]. 
5. Robustness of the discrete feedback control. From the definition of the discrete feedback $F$ and $F_{\mathbb{R}}$ it is obvious that these functions are typically discontinuous. Hence by applying this feedback law continuous dependence of the trajectories on the initial value will in general not hold.

This gives rise to the question of the robustness of the optimal trajectories. More precisely: Do optimal trajectories remain approximately optimal under small perturbations?

The answer is given in the following proposition and is essentially based on the Hoelder continuity of $v_{\delta}^{h}$ which satisfies

$$
\left|v_{\delta}^{h}(s, y)-v_{\delta}^{h}(\tilde{s}, \tilde{y})\right| \leq C\left(d_{\mathbb{S}}(s, \tilde{s})+d_{M}(y, \tilde{y})\right)^{\gamma}
$$

where $\gamma=\delta / L$ and $L$ is the Lipschitz constant of (3.1). For systems in $\mathbb{R}^{n}$ this immediately follows from [3, Lemma 4.1]; the proof is easily transferred to general manifolds. Here $d_{\mathbb{S}}$ and $d_{M}$ denote some metrics on $\mathbb{S}$ and $M$, respectively.

In what follows we allow time varying perturbations of the following kind: Assume that we have a time varying system on $\mathbb{S}^{d-1} \times K$ given by

$$
\begin{aligned}
& \dot{s}(t)=\tilde{h}(t, s(t), y(t), u(t)) \\
& \dot{y}(t)=\tilde{g}(t, y(t), u(t))
\end{aligned}
$$

with trajectories $\left(\tilde{s}\left(t, t^{*}, s_{0}, y_{0}, u(\cdot)\right), \tilde{y}\left(t, t^{*}, y_{0}, u(\cdot)\right)\right)$ using the initial time $t^{*}$. For some pair of initial values $\left(s_{0}, y_{0}\right)$ and a discrete Feedback $F$ with time step $h>0$ we denote the solution trajectories of (5.1) applying $F$ with initial time $t^{*}=0$ by $\left(\tilde{s}_{F}\left(t, s_{0}, y_{0}\right), \tilde{y}_{F}\left(t, y_{0}\right)\right)$. Using the abbreviations $t_{i}:=i h, \tilde{s}_{i}:=\tilde{s}_{F}\left(t_{i}, s_{0}, y_{0}\right), \tilde{y}_{i}:=$ $\tilde{y}_{F}\left(t_{i}, y_{0}\right)$ and $u_{i}:=F\left(\tilde{s}_{i}, \tilde{y}_{i}\right)$ we assume

$$
d_{\mathbb{S}}\left(\tilde{s}\left(t, t_{i}, \tilde{s}_{i}, \tilde{y}_{i}, u_{i}\right), s\left(t, \tilde{s}_{i}, \tilde{y}_{i}, u_{i}\right)\right)+d_{M}\left(\tilde{y}\left(t, t_{i}, \tilde{y}_{i}, u_{i}\right), y\left(t, \tilde{y}_{i}, u_{i}\right)\right)<\varepsilon_{i}
$$

for all $t \in[0, h]$, all $i \in \mathbb{N}$ and some sequence $\left(\varepsilon_{i}\right)_{i \in \mathbb{N}}$.

Proposition 5.1. Consider the system (3.1), a time step $h$, the corresponding optimal value function $v_{\delta}^{h}$ and the optimal discrete feedback $F$. Assume that a system (5.1) with the property (5.2) for some pair of initial values $(s, y)$ is given and denote the trajectories of (5.1) with initial time $t^{*}=0$ and the discrete feedback $F$ by $\left(\tilde{s}_{F}\left(t, s_{0}, y_{0}\right), \tilde{y}_{F}\left(t, y_{0}\right)\right)$.

Then for any $k \in \mathbb{N}$ the following inequality holds

$$
\left|v_{\delta}^{h}(s, y)-\tilde{J}_{\delta}(s, y, F)\right|<C \sum_{i=0}^{k-1} e^{-\delta h i} \varepsilon_{i}^{\gamma}+2 e^{-\delta h k} \frac{M_{q}}{\delta}
$$

where

$$
\tilde{J}_{\delta}(s, y, F):=\int_{0}^{\infty} e^{-\delta \tau} q\left(\tilde{s}_{F}(\tau, s, y), \tilde{y}_{F}(\tau, y), F\left(\tilde{s}_{F}\left(\left[\frac{\tau}{h}\right] h, s, y\right), \tilde{y}_{F}\left(\left[\frac{\tau}{h}\right] h, y\right) \tau\right)\right) d \tau
$$

is the value along the discrete feedback controlled trajectory of (5.1) and $M_{q}$ is the bound of $|q|$ on $\mathbb{S}^{d-1} \times K$.

Remark 5.2. Note that the right hand side of this inequality becomes small if the $\varepsilon_{i}$ are small for all sufficiently large $i \in \mathbb{N}$.

Proof. From the definition of $F$ and the assumption (5.2) it follows that

$$
\begin{aligned}
v_{\delta}^{h}(s, y) & =\int_{0}^{h} q\left(s_{F}(\tau, s, y), y_{F}(\tau, y), F(s, y)\right)+e^{-\delta h} v_{\delta}^{h}\left(s_{F}(h, s, y), y_{F}(h, y)\right) \\
& =\int_{0}^{h} q\left(\tilde{s}_{F}(\tau, s, y), \tilde{y}_{F}(\tau, y), F(s, y)\right)+e^{-\delta h} v_{\delta}^{h}\left(\tilde{s}_{F}(h, s, y), \tilde{y}_{F}(h, y)\right)+\tilde{C} \varepsilon_{0}^{\gamma}
\end{aligned}
$$


where $|\tilde{C}|<C$. On the other hand we obtain

$$
\tilde{J}_{\delta}(s, y, F)=\int_{0}^{h} q\left(\tilde{s}_{F}(\tau, s, y), \tilde{y}_{F}(\tau, y), F(s, y)\right)+e^{-\delta h} \tilde{J}_{\delta}\left(\tilde{s}_{F}(h, s, y), \tilde{y}_{F}(h, y), F\right)
$$

This yields

$$
\begin{aligned}
& \left|v_{\delta}^{h}(s, y)-\tilde{J}_{\delta}(s, y, F)\right| \\
& \quad \leq e^{-\delta h}\left|v_{\delta}^{h}\left(\tilde{s}_{F}(h, s, y), \tilde{y}_{F}(h, y)\right)-\tilde{J}_{\delta}\left(\tilde{s}_{F}(h, s, y), \tilde{y}_{F}(h, y), F\right)\right|+C \varepsilon_{0}^{\gamma}
\end{aligned}
$$

By observing that $v_{\delta}^{h}$ and $\tilde{J}_{\delta}$ are bounded by $M_{q} / \delta$ the assertion follows by induction.

This robustness property is the main tool for the linearization result in the next section.

6. Stabilization of the nonlinear system. We will now return to our original system (2.1). We recall the fact that $f(x, y, u)=A(y, u) x+\tilde{f}(x, y, u)$ where for $y$ in a compact set $K \subset M$ the estimate $\|\tilde{f}(x, y, u)\| \leq C_{f}\|x\|^{2}$ holds for all $x \in B_{\eta_{f}}(0)$, the ball with radius $\eta_{f}$ around $0, \mathrm{cp} .(2.3)$.

In analogy to Definition 3.2 we begin by defining the controllability concepts for system (2.1). Since we assume that the singular point $x^{*}$ coincides with the origin we may again formulate these concepts in terms of null controllability. As in the semilinear case we denote the exponential growth rates of a trajectory by

$$
\lambda_{f}^{t}\left(x_{0}, y_{0}, u(\cdot)\right):=\frac{1}{t} \ln \frac{\left\|x\left(t, x_{0}, y_{0}, u(\cdot)\right)\right\|}{\left\|x_{0}\right\|}
$$

and

$$
\lambda_{f}^{*}\left(x_{0}, y_{0}\right):=\inf _{u(\cdot) \in \mathcal{U}} \limsup _{t \rightarrow \infty} \lambda^{t}\left(x_{0}, y_{0}, u(\cdot)\right)
$$

DEFINITION 6.1. Let $K \subseteq M$ be a compact positively invariant set for the subsystem of (2.1) on $M$.

(i) The system (2.1) is called (locally) asymptotically null controllable over $K$ if there exists a neighbourhood $B(0)$ of 0 such that for any pair of initial values $\left(x_{0}, y_{0}\right) \in$ $B(0) \times K$ there exists a control function $u(\cdot) \in \mathcal{U}$ with

$$
\lim _{t \rightarrow 0}\left\|x\left(t, z_{0}, y_{0}, u(\cdot)\right)\right\|=0
$$

(ii) The system (2.1) is called (locally) exponentially null controllable over $K$ if there exists a neighbourhood $B(0)$ of 0 such that $\sup _{\left(x_{0}, y_{0}\right) \in B(0) \times K} \lambda_{f}^{*}\left(x_{0}, y_{0}\right)<0$.

(iii) The system (2.D) is called (locally) uniformly exponentially null controllable over $K$ if there exists a neighbourhood $B(0)$ of 0 and constants $C, \alpha>0$, such that for any pair of initial values $\left(x_{0}, y_{0}\right) \in B(0) \times K$ there exists a control function $u_{\left(x_{0}, y_{0}\right)}(\cdot) \in \mathcal{U}$ with

$$
\left\|z\left(t, x_{0}, y_{0}, u_{\left(x_{0}, y_{0}\right)}(\cdot)\right)\right\| \leq C e^{-\alpha t}|| x_{0} \|
$$

As in the semilinear case the implications (iii) $\Rightarrow$ (ii) $\Rightarrow$ (i) are obvious. However, for nonlinear systems the converse is not true, as the example below will show. Note 
that frequently the notion of exponential stability already demands the uniformity as in (iii), cf. e.g. [23] or [24].

We will now first prove some a-priori estimates for the solutions of (2.1) and (2.2).

LEMMA 6.2. Abbreviate with $(x(t), y(t))$ and $(z(t), y(t))$ the solutions of the systems (D.1) and (2.D) for a pair of initial values $\left(x_{0}, y_{0}\right)$ and a control function $u(\cdot)$. Let $T>0$ be a given time.

Then there exist constants $\alpha, \beta, C>0$ and $\eta(T)>0$ independent from $u(\cdot)$ such that for all $t \in[0, T]$ the following estimates hold:

(i) $\|x(t)\| \in\left[e^{-\alpha t}\left\|x_{0}\right\|, e^{\alpha t}\left\|x_{0}\right\|\right]$ for all $x_{0} \in B_{\eta(T)}(0)$

(ii) $\|z(t)\| \in\left[e^{-\alpha t}\left\|x_{0}\right\|, e^{\alpha t}\left\|x_{0}\right\|\right]$ for all $x_{0} \in \mathbb{R}^{d}$

(iii) $\|x(t)-z(t)\| \leq t C e^{\beta t}\left\|x_{0}\right\|^{2}$ for all $x_{0} \in B_{\eta}(T)(0)$

where $B_{\eta}(T)(0)$ denotes the ball with radius $\eta(T)$ around the origin.

Proof. (i) We show the estimate for the upper bound; the estimate for the lower bound follows from (ii) and (iii). From the linearization it follows that

$$
x(t)=x_{0}+\int_{0}^{t} A(y(\tau), u(\tau)) x(\tau)+\tilde{f}(x(\tau), y(\tau), u(\tau)) d \tau
$$

As long as $x(t) \in B_{\eta_{f}}(0)$ this implies

$$
\|x(t)\| \leq\left\|x_{0}\right\|+\int_{0}^{t} \alpha\|y(\tau)\| d \tau
$$

for some constant $\alpha>0$. This yields $\|x(t)\| \leq e^{\alpha t}\left\|x_{0}\right\|$ as long as $e^{\alpha t}|| x_{0} \| \leq \eta_{f}$ and hence the assertion follows with $\eta(T)=\eta_{f} / e^{\alpha T}$.

(ii) This is an easy consequence from the linearity of the system.

(iii) Define $m(t):=x(t)-z(t)$. From (i) and (ii) it follows that $\|m(t)\| \leq e^{\alpha t}\left\|x_{0}\right\|$. Furthermore $m$ is a solution of the differential equation

$$
\dot{m}(t)=A(y(t), u(t)) m(t)+\tilde{f}(y(s), m(t)+z(t), u(t)), z(0)=0
$$

and thus satisfies

$$
\begin{aligned}
\|m(t)\| & \leq \int_{0}^{t}\|A(y(\tau), u(\tau)) m(\tau)\|+\|\tilde{f}(y(\tau), m(\tau)+z(\tau), u(\tau))\| d \tau \\
& \leq \int_{0}^{t}\|A(y(\tau), u(\tau)) m(\tau)\|+C_{f}\left(\|m(\tau)\|^{2}+\|z(\tau)\|\|m(\tau)\|+\|z(\tau)\|^{2}\right) d \tau \\
& \leq t C_{f} e^{2 \alpha t}\left\|x_{0}\right\|^{2}+\int_{0}^{t} \gamma\|m(s)\| d s
\end{aligned}
$$

for some constant $\gamma>0$. Now the Gronwall Lemma yields

$$
\|m(t)\| \leq t C_{f} e^{2 \alpha t}\left\|x_{0}\right\|^{2} e^{\gamma t}
$$

and thus the assertion.

As in the semilinear case we may now write the exponential growth rate in finite time in integral form

$$
\lambda_{f}^{t}\left(x_{0}, y_{0}, u(\cdot)\right)=\frac{1}{t} \int_{0}^{t} q_{f}\left(x\left(\tau, x_{0}, y_{0}, u(\cdot)\right), y\left(\tau, y_{0}, u(\cdot)\right), u(\tau)\right) d \tau
$$


where

$$
q_{f}(x, y, u)=q\left(\frac{x}{\|x\|}, y, u\right)+\frac{x^{t} \tilde{f}(x, y, u)}{\|x\|^{2}}
$$

which can be calculated using the chain rule.

A simple calculation shows that

$$
\left\|x\left(t, x_{0}, y_{0}, u(\cdot)\right)\right\|=\left\|x_{0}\right\| e^{t \lambda_{f}^{t}\left(x_{0}, y_{0}, u(\cdot)\right) t}
$$

We can now apply the discrete feedback $F_{\mathbb{R}}$ from the previous sections to (2.1) by

$$
\begin{aligned}
& \dot{x}(t)=f\left(x(t), y(t), F_{\mathbb{R}}\left(x\left(\left[\frac{t}{h}\right] h\right), y\left(\left[\frac{t}{h}\right] h\right)\right)\right) \\
& \dot{y}(t)=g\left(y(t), F_{\mathbb{R}}\left(x\left(\left[\frac{t}{h}\right] h\right), y\left(\left[\frac{t}{h}\right] h\right)\right)\right)
\end{aligned}
$$

and denote the resulting trajectories by $\left(x_{F_{\mathrm{RR}}}\left(t, x_{0}, y_{0}\right), y_{F_{\mathrm{RR}}}\left(t, y_{0}\right)\right)$.

Defining the growth rate of $\left\|x_{F_{\mathbb{R}}}\left(t, x_{0}, y_{0}\right)\right\|$ in finite time by

$$
\lambda_{f}^{t}\left(x_{0}, y_{0}, F_{\mathbb{R}}\right):=\frac{1}{t} \ln \frac{\left\|x_{F_{\mathbb{R}}}\left(t, x_{0}, y_{0}\right)\right\|}{\left\|x_{0}\right\|}
$$

we obtain the following estimate.

Lemma 6.3. Let $\delta, h>0$ and let $F$ be an optimal discrete feedback with respect to $v_{\delta}^{h}$ for the linearization (2.2). Let $\sigma:=\sup _{(s, y) \in \mathbb{S}^{d-1} \times K} \delta v_{\delta}^{h}(x, y)$. Then for any $\varepsilon>0$ there exists an interval $\left[C_{-}(\varepsilon), C^{-}(\varepsilon)\right]$ and a constant $\eta(\varepsilon)>0$ such that for all pairs of initial values $x_{0}, y_{0}$ where $x_{0} \in B_{\eta(\varepsilon)}(0)$ the estimate

$$
\lambda_{f}^{t}\left(x_{0}, y_{0}, F_{\mathbb{R}}\right) \leq \sigma+\varepsilon
$$

holds for some $t \in\left[C_{-}(\varepsilon), C^{-}(\varepsilon)\right]$.

Proof. For a fixed pair of initial values $\left(x_{0}, y_{0}\right)$ and a control function $u(\cdot) \in \mathcal{U}$ we abbreviate $x(t):=x\left(t, x_{0}, y_{0}, u(\cdot)\right)$ and define

$$
\tilde{h}(t, s, y, u)=\frac{f(x(t), y, u)}{\|x(t)\|}-\left\langle\frac{f(x(t), y, u)}{\|x(t)\|}, s\right\rangle s
$$

for $s \in \mathbb{S}^{d-1}$. With $s_{0}:=x_{0} /\left\|x_{0}\right\|$ and $\tilde{s}\left(t, s_{0}, y_{0}, u(\cdot)\right):=x(t) /\|x(t)\|$ it follows that

$$
\dot{\tilde{s}}\left(t, s_{0}, y_{0}, u(\cdot)\right)=\tilde{h}\left(t, \tilde{s}\left(t, s_{0}, y_{0}, u(\cdot)\right), y\left(t, y_{0}, u(\cdot)\right), u(t)\right)
$$

hence the projection of the trajectory $x(t)$ onto $\mathbb{S}$ forms a solution trajectory of this time varying control system.

Let now $x_{i}:=x_{F_{\mathbb{R}}}\left(i h, x_{0}, y_{0}\right)$. Using Lemma 6.2 we obtain

$$
\left\|\frac{z\left(h, x_{i}, y_{i}, u\right)}{\left\|z\left(h, x_{i}, y_{i}, u\right)\right\|}-\frac{x\left(h, x_{i}, y_{i}, u\right)}{\left\|x\left(h, x_{i}, y_{i}, u\right)\right\|}\right\| \leq h C_{1}\left\|x_{i}\right\|
$$

By Lemma $6.2 x_{i}$ can be made arbitrarily small for each fixed $i \in \mathbb{N}$ by choosing $x_{0}$ sufficiently small and we can use Proposition 5.1 with $s=z /\|z\|$ and $\tilde{s}=x /\|x\|$ in order to obtain the estimate

$$
\delta \tilde{J}_{\delta}\left(x_{0}, y_{0}, F\right) \leq \sigma+\frac{\varepsilon}{4}
$$


for all sufficiently small $x_{0}$.

From the linearization estimates we obtain

$$
\left|\frac{x_{i}^{t} \tilde{f}\left(x_{i}, u\right)}{\left\|x_{i}\right\|^{2}}\right| \leq C_{f}\left\|x_{i}\right\| \leq C_{f} e^{\gamma t}|| x_{0} \|
$$

for all sufficiently small $\left\|x_{i}\right\|$, i.e. all sufficiently small $\left\|x_{0}\right\|$.

Hence $\left\|q\left(x_{F_{\mathbb{R}}}\left(t, x_{0}, y_{0}\right), \cdot, \cdot\right) /\right\| x_{F_{\mathbb{R}}}\left(t, x_{0}, y_{0}\right)\left\|-q_{f}\left(x_{F_{\mathbb{R}}}\left(t, x_{0}, y_{0}\right), \cdot, \cdot\right)\right\|$ can be made arbitrarily small on each bounded time interval by choosing $x_{0}$ sufficiently close to the origin and using [3, Lemma 4.1] we can conclude

$$
\delta \int_{0}^{\infty} e^{-\delta \tau} q_{f}\left(x_{F_{\mathbb{R}}}(\tau, x, y), y_{F_{\mathbb{R}}}(\tau, y), F_{\mathbb{R}}\left(x_{F_{\mathbb{R}}}\left(\left[\frac{\tau}{h}\right] h, x, y\right), y_{F_{\mathbb{R}}}\left(\left[\frac{\tau}{h}\right] h, y\right)\right)\right) d \tau \leq \sigma+\frac{\varepsilon}{2}
$$

for all sufficiently small $\left\|x_{0}\right\|$.

Now Lemma 3.5 (ii) yields the assertion.

In order prove the stability of (6.1) the last thing that remains to do is putting together the trajectory pieces.

Proposition 6.4. Consider system (2.1). Let $K \subseteq M$ be a compact positively invariant set for the subsystem of (2.1) on $M$. Assume that the linearization (2.D) is asymptotically null controllable over $K$. Then there is $\delta>0$ and $h>0$ such that the system (6.1) with the discrete feedback $F_{\mathbb{R}}$ is uniformly exponentially stable in some neighbourhood of the origin.

Proof. From the assumptions on the linearization Lemma 6.3 can be applied with $\sigma<0$.

Hence for all sufficiently small initial values $\left\|x_{0}\right\|$ there exists a $t \in\left[C_{-}(\varepsilon), C^{-}(\varepsilon)\right]$ such that

$$
\frac{1}{t} \ln \frac{\left\|x_{F_{\mathbb{R}}}\left(t, x_{0}, y_{0}\right)\right\|}{\left\|x_{0}\right\|} \leq \sigma+\varepsilon<0
$$

Abbreviating $x_{1}:=x_{F_{\mathbb{R}}}\left(t, x_{0}, y_{0}\right)$ it holds that $\left\|x_{1}\right\|<\left\|x_{0}\right\|$. Thus we can proceed inductively as in the proof of Proposition 3.3 and the assertion follows. $\square$

This proposition gives a characterization of exponential discrete feedback stabilizability by looking at its linearization. However, we would also like to have a characterization in terms of the nonlinear system itself. Clearly, since we are dealing with linearizations, asymptotic null controllability of the nonlinear system is not sufficient, see e.g. [4, Example (15)].

In fact, even exponential null controllability is not sufficient, as the following example shows. Consider

$$
\dot{x}=\left(\begin{array}{rr}
-1 & 0 \\
0 & 1
\end{array}\right) x+u_{1}\left(\begin{array}{rr}
-\frac{1}{2} & 0 \\
-\frac{1}{3} & \frac{1}{2}
\end{array}\right) x+u_{2}\left(\begin{array}{rr}
-\frac{1}{2} & 0 \\
\frac{1}{3} & \frac{1}{2}
\end{array}\right) x+u_{3}\left(\begin{array}{c}
x_{2}^{2} \\
0
\end{array}\right),
$$

where $U=[-1,1]^{3}$.

We claim that the linearized system is not asymptotically null controllable: Looking at the initial values $z_{0}=\left(0, z_{2}\right)^{T}, z_{2}>0$ it is easily seen that

$$
A(u) z_{0}=\left(\begin{array}{c}
0 \\
\left(1+\frac{1}{2} u_{1}+\frac{1}{2} u_{2}\right) z_{2}
\end{array}\right)
$$

Denoting the solution by $z\left(t, x_{0}, u(\cdot)\right)=\left(z_{1}\left(t, z_{0}, u(\cdot)\right), z_{2}\left(t, z_{0}, u(\cdot)\right)\right)^{T}$ we obtain $z_{1}\left(t, z_{0}, u(\cdot)\right) \equiv 0$ and $z_{2}\left(t, z_{0}, u(\cdot)\right) \geq z_{2}$ since $\left(1+\frac{1}{2} u_{1}(t)+\frac{1}{2} u_{2}(t)\right) \geq 0$ for all 
$u(\cdot) \in U$ and all $t \geq 0$. Thus we can conclude $\left\|z\left(t, z_{0}, u(\cdot)\right)\right\| \geq\left\|z_{2}\right\|$ for all $u(\cdot) \in \mathcal{U}$, meaning that for all initial values $z_{0}$ of the considered form no possible trajectory converges to the origin, which implies our claim.

However, for any $x=\left(x_{1}, x_{2}\right)^{T} \in \mathcal{C}$, where $\mathcal{C}$ is the cone defined by

$$
\mathcal{C}:=\left\{\left(\begin{array}{c}
x_{1} \\
x_{2}
\end{array}\right) \in \mathbb{R}^{2}|| \frac{x_{2}}{x_{1}} \mid<\frac{1}{10}\right\}
$$

we can choose the control $u_{x}=\left(u_{x 1}, u_{x 2}, u_{x 3}\right):=\left(\frac{2 x_{2}}{x_{1} / 3-x_{2}}, 0,0\right) \in U$. Then a simple computation yields

$$
f\left(x, u_{x}\right)=\left(-1-\frac{1}{2} u_{x 1}\right) x
$$

and since $u_{\alpha x}=u_{x}$ for all $\alpha \in \mathbb{R} \backslash\{0\}$ the solution for $u_{x}(\cdot) \equiv u_{x}$ satisfies

$$
x\left(t, x, u_{x}(\cdot)\right)=e^{\left(-1-\frac{1}{2} u_{x 1}\right) t} x .
$$

Hence the corresponding trajectory satisfies $\left\|x\left(t, x, u_{x}\right)\right\| \leq e^{-\frac{1}{2} t}|| x \|$ and thus converges to the origin exponentially fast.

For all initial values $x_{0} \in \mathbb{R}^{2} \backslash \mathcal{C}$ we choose $u(t) \equiv\left(-1,-1, \operatorname{sgn}\left(x_{1}\right)\right.$ ) (with the convention $\operatorname{sgn}(0)=1$ ) as long as the corresponding trajectory stays outside $\mathcal{C}$ and switch to $u_{x}$ from above once the trajectory reaches a point $x \in \mathcal{C}$.

Using this control function, any trajectory will enter the cone $\mathcal{C}$ in some finite time and then converge to the origin exponentially fast, thus the overall trajectory also converges to the origin exponentialy fast. Hence the nonlinear system is exponentially null controllable, although the semilinear system is not even asymptotically null controllable. Thus exponential null controllability of the nonlinear system does not imply asymptotic null controllability of the linearized system.

In order to formulate the desired result we therefore need the notion of uniform exponential null controllability.

Theorem 6.5. Consider system (2.1). Let $K \subseteq M$ be a compact positively invariant set for the subsystem of (2.1) on $M$. Then the following properties are equivalent:

(i) (2.1) is (locally) uniformly exponentially null controllable over $K$

(ii) (2.2) is asymptotically null controllable over $K$

(iii) There is $h>0$ and a discrete feedback that (locally) stabilizes (2.1) uniformly exponentially over $K$

Proof. "(ii) $\Rightarrow$ (iii)" is Proposition 6.4, "(iii) $\Rightarrow$ (i)" is immediately clear. It remains to show "(i) $\Rightarrow$ (ii)":

Let $B(0)$ be the neighbourhood in which uniform exponential null controllability holds. From (i) it follows that for any $\varepsilon>0$ there exists a $T>0$ such that for all $\left(x_{0}, y_{0}\right) \in B(0) \times K$ there exists a control function $u_{\left(x_{0}, y_{0}\right)}(\cdot) \in \mathcal{U}$ with

$$
\lambda_{f}^{T}\left(x_{0}, y_{0}, u_{\left(x_{0}, y_{0}\right)}(\cdot)\right)<-\alpha+\varepsilon
$$

Using the estimates from the proof of Lemma 6.3 we obtain for the growth rate of $(2.2)$

$$
\lambda^{T}\left(x_{0}, y_{0}, u_{\left(x_{0}, y_{0}\right)}(\cdot)\right)<-\alpha+2 \varepsilon
$$


for $x_{0}$ sufficiently close to the origin. Due to the linearity of (2.2) this estimate holds for all $x_{0} \in \mathbb{R}^{d}$. Now by induction we obtain the assertion as in the proof of Proposition 3.3.

This theorem shows in particular that any attempt to stabilize (2.1) at a singular point by using its linearization must fail if uniform exponential controllability is not satisfied, because the linearized system will not even be asymptotically null controllable. Conversely, exponential discrete feedback stabilization is always possible under this condition. We have therefore obtained the strongest result possible within the linearization approach.

A related result has been developed in [5] using Lyapunov functions: It is shown that for nonlinear systems asymptotic controllability to a (not necessarily singular) point $x$ implies stabilizability by means of a discrete feedback, where in order to reach $x$ the step size $h$ must tend to 0 . The result can therefore be interpreted as a kind of practical stabilization. In contrast to this practical stability here we obtain exponential stability using a discrete feedback with a fixed step size.

7. Conclusions. In this paper we developed results on the relation between null controllability and exponential stabilization by using a discrete feedback law for nonlinear systems at singular points. The construction of the feedback is obtained by minimizing the Lyapunov exponent of the linearized system, which forms a semilinear system. For semilinear systems asymptotic null controllability and exponential stabilizability by a discrete feedback turned out to be equivalent. For general nonlinear systems the equivalence between uniform exponential controllability and uniform exponential stabilizability has been shown. An example illustrated that uniform exponential controllability is in fact a necessary condition for the applicability of linearization techniques.

8. Appendix: Proof of Lemma 3.5. (i) Fix $\varepsilon>0$. We may assume $\sigma=-\varepsilon$ otherwise we use $q-\sigma-\varepsilon$ and $M_{q}+\sigma+\varepsilon$ instead of $q$ and $M_{q}$. Hence there exists $0 \leq T_{0}<T$ such that

$$
\int_{0}^{T_{0}} q(\tau) d \tau=-T_{0} \varepsilon \quad \text { and } \quad \int_{0}^{t} q(\tau) d \tau<-t \varepsilon \quad \text { for all } t>T_{0}
$$

This yields

$$
\int_{T_{0}}^{t} q(\tau) d \tau<\left(t-T_{0}\right)(-\varepsilon) \quad \text { for all } t>T_{0}
$$

Since for all $y \in[0,1)$ the inequality $\ln (1-y) \leq-y$ and hence $e^{-y} \geq 1-y$ holds, we obtain

$$
\begin{aligned}
\left|\int_{0}^{T_{0}} q(\tau) d \tau-\int_{0}^{T_{0}} e^{-\delta t} q(\tau) d \tau\right| & \leq T_{0}\left(1-e^{-\delta T_{0}}\right) M \\
& \leq T\left(1-e^{-\delta T}\right) M \\
& \leq \delta T^{2} M
\end{aligned}
$$

Thus the inequality in (8.1) implies for $\delta<\frac{\varepsilon}{M T}<\frac{\varepsilon}{M T_{0}}$

$$
\delta \int_{0}^{T_{0}} e^{-\delta \tau} q(\tau) d \tau<\delta\left(-T_{0} \varepsilon+\delta T^{2} M\right)<0
$$


Now fix $\tilde{\varepsilon}>0$. Since $q$ is bounded there exists $T_{1}>T_{0}$ such that

$$
\left|\int_{T_{0}}^{\infty} e^{-\delta \tau} q(\tau) d \tau-\int_{T_{0}}^{T_{1}} e^{-\delta \tau} q(\tau) d \tau\right| \leq \tilde{\varepsilon}
$$

From estimate $(8.2)$ choose $\gamma>0$ maximal with the property

$$
\int_{T_{0}}^{T_{1}} q^{+}(\tau) d \tau-\int_{T_{0}}^{T_{1}-\gamma} q^{-}(\tau) d \tau=0
$$

where $q^{+}$and $q^{-}$denote the positive and negative part of $q$, respectively. Now we can define a monotonically decreasing sequence $\tau_{i}, i \in \mathbb{N}$ by $\tau_{1}:=T_{1}, \tau_{2}:=T_{1}-\gamma$, and

$$
\tau_{i+1}:=\min \left\{t \in\left[T_{0}, \tau_{i}\right] \mid-\int_{t}^{\tau_{i}} q^{-}(\tau) d \tau+\int_{\tau_{i}}^{\tau_{i-1}} q^{+}(\tau) d \tau=0\right\}
$$

This sequence is well defined: Assume that there exists $\tau_{i}$ for some $i \geq 2$. In the case $i>2$ for all $j$ with $i \geq j \geq 3$ the equality

$$
-\int_{T_{0}}^{\tau_{j}} q^{-}(s) d s+\int_{\tau_{j}}^{\tau_{j-1}} q^{+}(s) d s=-\int_{T_{0}}^{\tau_{j-1}} q^{-}(s) d s+\int_{\tau_{j-1}}^{\tau_{j-2}} q^{+}(s) d s
$$

holds and by induction and the choice of $\gamma$ in (8.5) it follows

$$
-\int_{T_{0}}^{\tau_{i}} q^{-}(\tau) d \tau+\int_{\tau_{i}}^{\tau_{i-1}} q^{+}(\tau) d \tau=-\int_{T_{0}}^{T_{1}-\gamma} q^{-}(\tau) d \tau+\int_{T_{1}-\gamma}^{T_{1}} q^{+}(\tau) d \tau \leq 0
$$

This guarantees the existence of $\tau_{i+1}$. Since $\left(\tau_{i}\right)$ is monotone and bounded the sequence converges to some $\tilde{\tau} \geq T_{0}$. We claim $\tilde{\tau}=T_{0}$ :

By the definition of $\tau_{i}$ it follows that $-\int_{\tau_{i+1}}^{T_{1}-\gamma} q^{-}(\tau) d \tau+\int_{\tau_{i}}^{T_{1}} q^{+}(\tau) d \tau=0$. The convergence $\tau_{i} \rightarrow \tilde{\tau}$ yields the equality

$$
-\int_{\tilde{\tau}}^{T_{1}-\gamma} q^{-}(\tau) d \tau+\int_{\tilde{\tau}}^{T_{1}} q^{+}(\tau) d \tau=0
$$

This implies $\int_{T_{0}}^{\tilde{\tau}} q(\tau) d \tau=0$, which shows the asserted equality using (8.2).

Hence we can choose $k \in \mathbb{N}$ such that $\left|\tau_{k-1}-T_{0}\right| \leq \tilde{\varepsilon}$ and replace $\tau_{k}$ by $\tau_{k}=T_{0}$. Thus we can estimate

$$
\begin{aligned}
\int_{T_{0}}^{T_{1}} e^{-\delta \tau} q(\tau) d \tau & \leq \sum_{i=2}^{k-1}\left(-\int_{\tau_{i+1}}^{\tau_{i}} e^{-\delta \tau} q^{-}(\tau) d \tau+\int_{\tau_{i}}^{\tau_{i-1}} e^{-\delta \tau} q^{+}(\tau) d \tau\right)+M \tilde{\varepsilon} \\
& \leq \sum_{i=2}^{k-1} \underbrace{\left(-\int_{\tau_{i+1}}^{\tau_{i}} e^{-\delta \tau_{i}} q^{-}(\tau) d \tau+\int_{\tau_{i}}^{\tau_{i-1}} e^{-\delta \tau_{i}} q^{+}(\tau) d \tau\right)}_{=0}+M \tilde{\varepsilon} \\
& =M \tilde{\varepsilon}
\end{aligned}
$$

In connection with (8.3) and (8.4) this yields

$$
\int_{0}^{\infty} e^{-\delta \tau} q(\tau) d \tau=\int_{0}^{T_{0}} e^{-\delta \tau} q(\tau) d \tau+\int_{T_{0}}^{T_{1}} e^{-\delta \tau} q(\tau) d \tau+\int_{T_{1}}^{\infty} e^{-\delta \tau} q(\tau) d \tau<0+M \tilde{\varepsilon}+\tilde{\varepsilon}
$$


Since $\tilde{\varepsilon}>0$ was arbitrary this proves (i).

(ii) Assume the opposite: Let

$$
\frac{1}{t} \int_{0}^{t} q(\tau) d \tau>\sigma+\varepsilon \text { for all } t \in\left[\frac{\varepsilon}{(4 M+4 \sigma+\varepsilon) \delta},-\frac{\ln \frac{\varepsilon}{4 M}}{\delta}\right]
$$

We define $\tilde{q}$ via

$$
\tilde{q}(\tau):= \begin{cases}q(\tau), & \tau \leq-\frac{\ln \frac{\varepsilon}{4 M}}{\delta} \\ \sigma+\varepsilon, & \tau>-\frac{\ln \frac{\varepsilon}{4 M}}{\delta}\end{cases}
$$

This yields

$$
\frac{1}{t} \int_{0}^{t} \tilde{q}(\tau) d \tau>\sigma+\varepsilon \text { for all } t \geq \frac{\varepsilon}{(4 M+4 \sigma+\varepsilon) \delta}
$$

and by (i) (with opposite signs and inequalities) we obtain

$$
\delta \int_{0}^{\infty} e^{-\delta \tau} \tilde{q}(\tau) d \tau \geq \sigma+\frac{3}{4} \varepsilon
$$

Hence

$$
\begin{aligned}
\delta \int_{0}^{\infty} e^{-\delta \tau} q(\tau) d \tau & =\delta \int_{0}^{\infty} e^{-\delta \tau} \tilde{q}(\tau) d \tau-\delta \int_{-\frac{\ln \frac{\varepsilon}{4 M}}{\delta}}^{\infty} \tilde{q}(\tau)-q(\tau) d \tau \\
& \geq \sigma+\frac{3}{4} \varepsilon-\delta \int_{-\frac{\ln \frac{\varepsilon}{4 L}}{\delta}}^{\infty} e^{-\delta \tau} 2 M d \tau=\sigma+\frac{1}{4} \varepsilon
\end{aligned}
$$

which contradicts the assumption on this discounted integral.

(iii) By (ii) for any $\varepsilon>0$ there exist times $\tau(t)$ bounded from below and above such that

$$
\frac{1}{\tau(t)} \int_{0}^{\tau(t)} q(t+\tau) d \tau<\sigma+\varepsilon
$$

Hence $\tau_{0}:=0, \tau_{i+1}=\tau_{i}+\tau\left(\tau_{i}\right)$ defines a monotonically increasing sequence diverging to infinity for which there exists $a \in \mathbb{R}$ such that $\tau_{i+1}-\tau_{i}<a$ for all $i \in \mathbb{N}$. For arbitrary $T>0$ let $\tau_{i(T)}$ be the maximal element of this sequence satisfying $\tau_{i(T)} \leq T$. Thus we obtain

$$
\begin{aligned}
\limsup _{T \rightarrow \infty} \frac{1}{T} \int_{0}^{T} q(\tau) d \tau & =\limsup _{T \rightarrow \infty} \frac{1}{T}\left(\sum_{j=0}^{i(T)} \int_{\tau_{j-1}}^{\tau_{j}} q(\tau) d \tau+\int_{\tau_{i(T)}}^{T} q(\tau) d \tau\right) \\
& \leq \limsup _{T \rightarrow \infty}\left(\sigma+\varepsilon+\frac{a M}{T}\right)=\sigma+\varepsilon
\end{aligned}
$$

Since $\varepsilon>0$ was arbitrary the assertion follows. $\square$

\section{REFERENCES}

[1] P. Cannarsa and H. Frankowska, Some characterizations of optimal trajectories in control theory, SIAM J. Control Optim., 29 (1991), pp. 1322-1347. 
[2] I. Cafuzzo Dolcetta, On a discrete approximation of the Hamilton-Jacobi equation of dynamic programming, Appl. Math. Optim., 10 (1983), pp. 367-377.

[3] I. Cafuzzo Dolcetta and H. Ishit, Approximate solutions of the Bellman equation of deterministic control theory, Appl. Math. Optim., 11 (1984), pp. 161-181.

[4] R. Chabour, G. Sallet, and J. Vivalda, Stabilization of nonlinear systems: A bilinear approach, Math. Control Signals Syst., 6 (1993), pp. 224-246.

[5] F. Clarke, Y. Ledyaev, E. Sontag, and A. Subbotin, Asymptotic controllability implies feedback stabilization, IEEE Trans. Autom. Control. To appear.

[6] F. Colonius and W. Kliemann, Linear control semigroups acting on projective space, J. Dyn. Differ. Equations, 5 (1993), pp. 495-528.

[7] - Maximal and minimal Lyapunov exponents of bilinear control systems, J. Differ. Equations, 101 (1993), pp. 232-275.

[8] - Asymptotic null controllability of bilinear systems, in "Geometry in Nonlinear Control and Differential Inclusions", B. Jakubczyk and W. Respondek, eds., Banach Center Publications Vol. 32, Warsaw, 1995, pp. 139-148.

[9] M. FALCONE, A numerical approach to the infinite horizon problem of deterministic control theory, Appl. Math. Optim., 15 (1987), pp. 1-13. Corrigenda, ibid. 23 (1991), 213-214.

[10] H. FranKowsKa, Optimal trajectories associated with a solution of the contingent HamiltonJacobi Equation, Appl. Math. Optim., 19 (1989), pp. 291-311.

[11] L. GRÜne, Discrete feedback stabilization of semilinear control systems, ESAIM: Control, Optimisation and Calculus of Variations, 1 (1996), pp. 207-224.

[12] - Numerical stabilization of bilinear control systems, SIAM J. Control Optim., 34 (1996), pp. 2024-2050.

[13] - An adaptive grid scheme for the discrete Hamilton-Jacobi-Bellman equation, Numer. Math., 75 (1997), pp. 319-337.

[14] - Discrete feedback stabilization of nonlinear control systems at a singular point, in Proceedings of the 4th European Control Conference, Brussels, 1997. Paper No. 806.

[15] W. Hahn, Stability of Motion, Springer-Verlag Berlin, Heidelberg, 1967.

[16] H. Hermes, On stabilizing feedback attitude control, J. Optimization Theory Appl., 31 (1980), pp. 373-384.

[17] - On the synthesis of stabilizing feedback control via Lie algebraic methods, SIAM J. Control Optim., 18 (1980), pp. 352-361.

[18] N. N. Krasovskĭ and A. I. SubBotin, Game-Theoretical Control Problems, Springer Verlag, New York, 1988.

[19] P. L. Lions, Generalized solutions of Hamilton-Jacobi equations, Pitman, London, 1982.

[20] E. D. SonTAg, Nonlinear regulation: The piecewise linear approach, IEEE Trans. Autom. Control, AC-26 (1981), pp. 346-358.

[21] — Mathematical Control Theory, Springer Verlag, New York, 1990.

[22] - Feedback stabilization using two-hidden-layer nets, IEEE Trans. Neural Networks, 3 (1992), pp. 981-990.

[23] M. Vidyasagar, Nonlinear System Analysis, Prentice-Hall, Englewood Cliffs, New Jersey, 1993.

[24] J. ZABCZYK, Some comments on stabilizability, Appl. Math. Optim., 19 (1989), pp. 1-9. 